\title{
Nonlinear fiber gyroscope for quantum metrology
}

\author{
Alfredo Luis* and Irene Morales \\ Departamento de Óptica, Facultad de Ciencias Físicas, Universidad Complutense, 28040 Madrid, Spain
}

Ángel Rivas

Departamento de Física Teórica I, Facultad de Ciencias Físicas, Universidad Complutense, 28040 Madrid, Spain

(Received 14 February 2016; published 18 July 2016)

\begin{abstract}
We examine the performance of a nonlinear fiber gyroscope for improved signal detection beating the quantum limits of its linear counterparts. The performance is examined when the nonlinear gyroscope is illuminated by practical field states, such as coherent and quadrature squeezed states. This is compared with the case of more ideal probes such as photon-number states.
\end{abstract}

DOI: 10.1103/PhysRevA.94.013830

\section{INTRODUCTION}

Signal-detection strategies based on nonlinear processes can clearly outperform current strategies based on linear processes [1-13]. This is so even when using probes in classical-like states. This is relevant because classical-like states are characterized by their robustness against practical imperfections, which can be deadly for schemes using probes prepared in nonclassical states [14-16].

A suitable arena for nonlinear detection schemes is optics. The most precise detection schemes are optical interferometers, and nonlinear processes are quite simply implemented in optics via propagation in nonlinear media.

In this work we focus on the quantum limits to the resolution achievable in gyroscopes as good candidates to exploit the benefits of nonlinear detection, paying special attention to quantum characteristics exclusive of this interferometer [17]. We will focus on the case when the gyroscope is illuminated by practical field states, i.e., that can be generated in practice and are robust against imperfections, such as coherent and quadrature squeezed states. For the sake of comparison the results will be compared with the case of probes in less practical states, such as product of number states.

There are two important advantages in the proposed scheme with respect to other nonlinear detection schemes, namely, its improved optical performance and its capability to measure angles. More specifically, as a comparison with other interferometers (like Michelson's), nonlinearity is integrated as a constituent part of the gyroscope, so that it is not necessary to modify the setup to include extra elements to provide the nonlinear effect. For instance, in order to include nonlinearity in the LIGO gravitational-wave detector we should "attach" some nonlinear material to it [18]. The simplest way to do this would be by filling the room with a nonlinear gas. This will produce severe practical inconvenience, since gases are a reported source of technical noise because of density fluctuations and light scattering, which is the reason why LIGO works at ultra-high vacuum. Furthermore, the nonlinearity in gases is typically small. An alternative would be to attach

\footnotetext{
*alluis@ fis.ucm.es; http://www.ucm.es/info/gioq
}

a piece of nonlinear material to the suspended masses. In such a case some light will be reflected in the interphase, and in any case it is far from technically simple to make such attachments because of the large dimensions of the device. Our proposal is free of these problems because the nonlinearity is automatically embedded as a part of the interferometer: the optical fiber. The optical performance of fiber glasses is much superior to gases regarding homogeneities and any other source of scattering and fluctuations. Moreover, the nonlinear effect is much larger than for gases, being improved by the very large electric fields that can be reached by light confinement in the small volumes of the fiber core. Gyroscopes do not need to be kilometer-long devices to provide extremely long optical paths required for precision interferometry since looped fiber-optics coil multiplies the length and the cumulative effects of nonlinearlity by the number of loops. Finally, the gyroscope is a rigid detector without moving parts, that always provides a more robust and improved optical performance.

Here we consider applying nonlinear quantum metrology in a gyroscopic scheme. This is very timely because there are many interesting unobserved effects caused by rotations, specially to test gravitational theories and phenomena. For example, the Lense-Thirring effect, a relativistic effect not observed yet, the local space-time curvature, or the existence of a preferred frame in the Universe [19].

Besides fiber-optics realizations, previous works have shown that nonlinear interferometers are feasible in other physical contexts, such as Bose-Einstein condensates [3-5,7,20] and nanomechanical resonators [21]. Finally, we may point out that gyroscopes share geometry with sensors sensible to physical variables different from rotations and involving relevant physical phenomena such as the Aharonov-Bohm effect, for example [22].

\section{MODEL}

Any detection scheme involves four steps. In the first one, some probe state $|\psi\rangle$ is prepared. In the second step the probe experiences a signal-dependent transformation $U(\phi)$. Then, the measurement of some observable $M$ is performed at the output state of the probe $U(\phi)|\psi\rangle$. With the results of the measurement the signal $\phi$ and its uncertainty $\Delta \phi$ are estimated. 


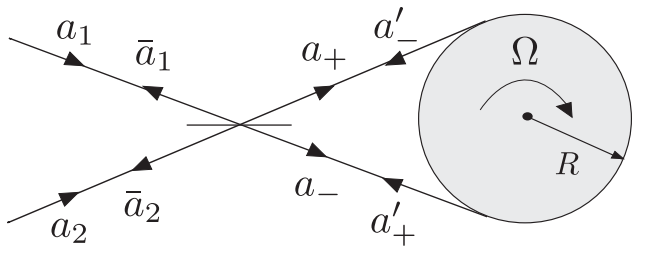

FIG. 1. Scheme of the gyroscope illustrating the definition of the field modes.

\section{A. Probe and system modes}

The probe $|\psi\rangle$ is the light state illuminating the gyroscope. Within the interferometer the system is made of two counterpropagating modes with complex-amplitude operators $a_{ \pm}$, that can be feed trough a lossless $50 \%$ beam splitter coupling the inner modes $a_{ \pm}$with two input modes $a_{1,2}$ (see Fig. 1),

$$
a_{ \pm}=\frac{1}{\sqrt{2}}\left(a_{1} \mp i a_{2}\right) \text {. }
$$

The probes are prepared in modes $a_{1,2}$ while the observation will be made at the output modes $\bar{a}_{1,2}$ leaving the gyroscope. These are related to the modes within the interferometer through a beam splitter performing the transformation inverse to the one in Eq. (2.1),

$$
\bar{a}_{1}=\frac{1}{\sqrt{2}}\left(a_{+}^{\prime}+a_{-}^{\prime}\right), \quad \bar{a}_{2}=\frac{i}{\sqrt{2}}\left(a_{+}^{\prime}-a_{-}^{\prime}\right),
$$

where $a_{ \pm}^{\prime}$ are the amplitudes at the end of the nonlinear fiber, while $a_{ \pm}$refer to the amplitudes at the beginning.

\section{B. Signal-dependent transformation}

In the second step the probe experiences a signal-dependent transformation $U(\phi)$. The rotation of the gyroscope introduces an asymmetry between the times spent by the two modes $a_{ \pm}$ within the interferometer, which leads to a phase-difference,

$$
\varphi \simeq \frac{\omega}{c} L\left(n_{+}-n_{-}\right)+2 \frac{\omega A \mathcal{N}}{c^{2}} \Omega\left(n_{+}^{2}+n_{-}^{2}\right),
$$

where $\omega$ is the field frequency, $L$ is the length of the fiber, $A=\pi R^{2}$ is the area enclosed by a single loop of the fiber made of loops of radius $R, \mathcal{N}$ is the number of loops, $\Omega$ is the angular speed, $n_{ \pm}$are the indices of refraction for the corresponding modes, and we have assumed that the speed acquired by the fiber due to $\Omega$ is much smaller than the speed of light in vacuum $c$.

The key point for our work is that we are dealing with nonlinear media so the indices of refraction $n_{ \pm}$depend on the light intensities. Assuming Kerr-type nonlinearity and light traveling as pulses of frequency $\omega$, cross-section $\mathcal{A}$, and duration $\tau$, carrying a number of photons $N_{ \pm}$, we will have

$$
n_{ \pm}^{2} \simeq n_{0}^{2}+\chi_{ \pm} E_{ \pm}^{2} \simeq n_{0}^{2}+\frac{\mu_{0} \hbar \omega c \chi_{ \pm}}{\mathcal{A} \tau} N_{ \pm},
$$

where $n_{0}$ is the linear index, $\chi_{ \pm}$are the nonlinear susceptibilities, for simplicity the medium is assumed optically isotropic so that the linear index is the same for both modes, $E_{ \pm}$are the electric-field strengths, and $\mu_{0}$ is the magnetic permeability of the vacuum. The second equality in Eq. (2.4) is just a rough approximation to motivate the ongoing quantum analysis. We have assumed that there are no crossed terms. This is specially so in a pulsed illumination since in such a case the overlap of the counter propagating pulses is negligible.

In the quantum analysis, the propagation of the modes $a_{ \pm}$ within the fiber can be described by the unitary operator $U_{\Omega} U_{0}$, where $U_{\Omega}$ includes all the signal-dependent effects given by the second term on the right-hand side of Eq. (2.3), while $U_{0}$ contains the contributions independent of $\Omega$, this is the contribution of the first term on the right-hand side of Eq. (2.3). The factorization is possible because both parts can be fully expressed in terms of two commuting phase shifts generated by different powers of the photon-number operators.

In this work we focus on the nonlinear part of the signaldependent component $U_{\Omega}$. The contribution by $U_{0}$ will be invoked just to introduce and additional fixed phase $\phi_{0}$ when necessary. Otherwise, it will be assumed embodied in the probe preparation or compensated by a similar amount of fiber propagation not experiencing the rotation.

For the signal-dependent part $U_{\Omega}$ we will just consider the nonlinear contribution given by the second term on the righthand side of Eq. (2.4). This should be the dominating part for sufficiently large photon numbers, as far as we intend to exploit the asymptotic behavior allowed by nonlinear effects. In any case, the phase shifts produced by the linear and nonlinear parts of the transformation may be addressed simultaneously via multiparameter estimation procedure [23]. This is studied in more detail in Appendix B showing that it agrees with the expected results both for the linear and nonlinear parts.

Thus, the signal-dependent transformation we are going to study is

$$
U_{\Omega}=e^{-i \phi G}, \quad G=N_{+}^{2}-N_{-}^{2},
$$

where $N_{ \pm}=a_{ \pm}^{\dagger} a_{ \pm}$are the corresponding number operators and the signal takes the form $\phi=\mu_{0} A \hbar \omega^{2} \mathcal{N} \chi \Omega /(\mathcal{A} \tau c)$, assuming the nonlinear susceptibilities identical for both modes $\chi_{+}=\chi_{-}=\chi$. Note that the relative sign in Eq. (2.5) is the correct one for counter-propagating modes. The sing depending on the propagation direction is often expressed by saying that for counterpropagating modes the generator $G$ is proportional to momentum rather than to energy [24].

Using Eq. (2.1) we find an useful expression for the generator $G$, in terms of the input modes $a_{1,2}$ by

$$
G=N\left(N_{+}-N_{-}\right)=i N\left(a_{2}^{\dagger} a_{1}-a_{1}^{\dagger} a_{2}\right),
$$

where $N$ is the total-number operator

$$
N=N_{+}+N_{-}=N_{1}+N_{2}
$$

and we have used that $N$ is a conserved quantity at the beam splitter. We note that the second equality in Eq. (2.6) is formally the same generator already tested experimentally in Ref. [10] in a very different context.

\section{Measurement}

The most simple measurement sensitive to signals encoded as phase shifts is the interference achieved by the coupling at the beam splitter of the $a_{ \pm}$modes after the nonlinear propagation, $a^{\prime}=U_{\Omega}^{\dagger} a_{ \pm} U_{\Omega}$, followed by photon-number detection at the outgoing beams $\bar{a}_{1,2}$. This provides us with a 
photon-number statistics $p\left(n_{1}, n_{2} \mid \phi\right)$ containing the complete information about the signal available in this arrangement. In order to extract this information the most simple option is to consider the difference between the output photon numbers as $M=\bar{a}_{1}^{\dagger} \bar{a}_{1}-\bar{a}_{2}^{\dagger} \bar{a}_{2}$. Since the transformation Eq. (2.5) is quite simple, we have the following expression for $M$ in terms of the complex amplitudes at the beginning of the fiber $a_{ \pm}$:

$$
M=a_{+}^{\dagger} e^{i \Lambda} a_{-}+a_{-}^{\dagger} e^{-i \Lambda} a_{+}, \quad \Lambda=2 \phi N+\phi_{0},
$$

and $\phi_{0}$ is any fixed additional linear phase shift introduced to optimize performance. Then, we can express $M$ in terms of the input modes $a_{1,2}$ via Eq. (2.1) as

$$
M=a_{1}^{\dagger} C a_{1}-a_{2}^{\dagger} C a_{2}-a_{2}^{\dagger} S a_{1}-a_{1}^{\dagger} S a_{2},
$$

where $C=\cos \Lambda, S=\sin \Lambda$.

For the most simple situations the lowest-order moments of $M$ may be enough. However, there are situations where they may not extract most of the signal information encoded in the output field state. In such situations we may look at the complete output photon-number statistics $p\left(n_{1}, n_{2} \mid \phi\right)$ in order to better understand the situation.

\section{Simple estimation}

The key performance estimator is the signal uncertainty $\Delta \phi$. In a very simple first approach this can be estimated from the lowest-order moments of $M$ via the signal to noise ratio, or, equivalently, from a simple error propagation as

$$
\Delta \phi=\frac{\Delta M}{|\partial\langle M\rangle / \partial \phi|}=\frac{\Delta M}{|\langle[M, G]\rangle|} \geqslant \frac{1}{2 \Delta G},
$$

where in the last inequality the uncertainty relation $\Delta G \Delta M \geqslant$ $\mid\langle[G, M]\rangle / 2$ has been used. In some relevant situations optimum results are obtained if $\phi_{0}=-\pi / 2$ so that for very small signals $\langle M\rangle$ will be near zero, which is the point of maximum sensitivity to phase variations. In such a case for $\phi \rightarrow 0$ we have after Eqs. (2.9), (2.5), and (2.6),

$$
M=i\left(a_{-}^{\dagger} a_{+}-a_{+}^{\dagger} a_{-}\right)=a_{1}^{\dagger} a_{2}+a_{2}^{\dagger} a_{1},
$$

and

$$
[M, G]=2 i N\left(a_{+}^{\dagger} a_{-}+a_{-}^{\dagger} a_{+}\right)=2 i\left(N_{1}^{2}-N_{2}^{2}\right) .
$$

At this stage one might be tempted to look for optimum results in terms of minimum uncertainty states of $G$ and $M$, granting the equality in the last step in Eq. (2.10). However, this is not quite so optimum strategy since some other probes may lead to smaller $\Delta \phi$ via a larger $\Delta G$ even though they are not minimum [25].

\section{E. Advanced estimation}

The estimation of the uncertainty $\Delta \phi$ can be addressed using more powerful tools such as the Cramér-Rao lower bound and the quantum Fisher information $F_{Q}$ as [26]

$$
\Delta \phi \geqslant \frac{1}{\sqrt{F}} \geqslant \frac{1}{\sqrt{F_{Q}}} \geqslant \frac{1}{2 \Delta G},
$$

where $F$ is the Fisher information

$$
F=\sum_{n=0}^{\infty} \frac{1}{p_{n}}\left(\frac{\partial p_{n}}{\partial \phi}\right)^{2},
$$

with $p_{n} \equiv p\left(n_{1}, n_{2} \mid \phi\right)$ and $n$ standing for the pair of natural numbers $\left(n_{1}, n_{2}\right)$ representing the number of photons registered at the two outputs of the gyroscope. In the case of no additional phase shift $\phi_{0}=0$, the output photon-number statistics is

$$
\begin{aligned}
p_{n} & =\left|\left\langle n_{1}, n_{2}\left|U_{B S}^{\dagger} e^{-i \phi G} U_{B S}\right| \psi\right\rangle\right|^{2} \\
& =\left|\left\langle n_{1}, n_{2}\left|e^{\phi N\left(a_{2}^{\dagger} a_{1}-a_{1}^{\dagger} a_{2}\right)}\right| \psi\right\rangle\right|^{2},
\end{aligned}
$$

where $U_{\mathrm{BS}}$ is the unitary operator representing the action of the input beam splitter, $\left|n_{1}, n_{2}\right\rangle$ are number states, and we have taken into account that the output beam splitter performs the inverse transformation of the input.

In the case that the probe is in a pure state with real coefficients in the number basis $\left\langle n_{1}, n_{2} \mid \psi\right\rangle \in \mathbb{R}$, then $p_{n}=c_{n}^{2}$, with $c_{n}=\left\langle n_{1}, n_{2}\left|e^{\phi N\left(a_{2}^{\dagger} a_{1}-a_{1}^{\dagger} a_{2}\right)}\right| \psi\right\rangle \in \mathbb{R}$ and it can be easily shown that [27]

$$
F=\sum_{n=0}^{\infty} 4\left(\frac{\partial c_{n}}{\partial \phi}\right)^{2}=4 \Delta^{2} G=F_{Q},
$$

where in the last step we have used that if $\left\langle n_{1}, n_{2} \mid \psi\right\rangle \in \mathbb{R}$ then $\langle\psi|G| \psi\rangle=0$ after Eq. (2.6).

Naturally, the fact that the full photon number statistics outperforms those of the simple measurement of $M$ is rather obvious since the statistics of $M$ is a marginal of the full statistics $p_{n}$. The key point here is that when the probe state has real coefficients in the number basis, the $p_{n}$ statistics contains all the information conveyed by the transformed probe state so that its Fisher information equals the quantum Fisher information. Note that this conclusion holds for all $\phi$. Since the total-number variable $N$ does not provide phase information, all the phase information in $p_{n}$ is actually provided by $M$. As we shall see, in many cases of interest the two lowest-order moments of $M$ already contain all the relevant information about $\phi$.

\section{QUANTUM RESOLUTION FOR DIFFERENT PROBES}

Typically, $\Delta \phi$ decreases as the mean number of photons $\bar{N}$ in the probe state $|\psi\rangle$ increases. So the usual task is to look for the minimum $\Delta \phi$ at fixed $\bar{N}$, this is to say, minimum uncertainty at fixed-energy resources. Alternatively, this is to inquire about the probe states that provide the best scaling of $\Delta \phi$ as a function of $\bar{N}$. To this end we will consider different probe states under the two estimation uncertainties in Secs. II D and IIE.

\section{A. Product of coherent states}

If the input probe is in a product of Glauber coherent states $|\psi\rangle=\left|\alpha_{1}\right\rangle\left|\alpha_{2}\right\rangle$, the field state in modes $a_{ \pm}$will be as well a product of coherent states $|\psi\rangle=\left|\alpha_{+}\right\rangle\left|\alpha_{-}\right\rangle$with the same total mean number of photons $\bar{N}=\left|\alpha_{+}\right|^{2}+\left|\alpha_{-}\right|^{2}=\left|\alpha_{1}\right|^{2}+\left|\alpha_{2}\right|^{2}$. A simple calculus leads exactly to

$$
\langle M\rangle=2 \sqrt{\bar{n}_{+} \bar{n}_{-}} e^{-2 \bar{N} \sin ^{2} \phi} \cos \left[\bar{N} \sin (2 \phi)+\phi_{0}\right],
$$


while

$$
\begin{aligned}
\left\langle M^{2}\right\rangle= & \bar{N}+2 \bar{n}_{+} \bar{n}_{-}(1 \\
& \left.+e^{-2 \bar{N} \sin ^{2}(2 \phi)} \cos \left[\bar{N} \sin (4 \phi)+4 \phi+2 \phi_{0}\right]\right),
\end{aligned}
$$

where $\bar{n}_{ \pm}=\left|\alpha_{ \pm}\right|^{2}$ and we have assumed real $\alpha_{ \pm}$. Typically, $\phi$ is small enough so that $\sin \phi \simeq \phi$ and $\sin (2 \phi) \simeq 2 \phi$. Moreover, will take advantage of the robustness of coherent states to consider a large mean number of photons $\bar{N} \gg 1$.

We can observe that the dispersion of total number of photons in the coherent state degrades the visibility of the interference through the factor $e^{-2 \bar{N} \phi^{2}}$. Therefore, in order to obtain meaningful results it is convenient to assume that $\sqrt{\bar{N}} \phi \ll 1$. This is to say that the signal expected is below the standard quantum limit, so it would pass unnoticed if the fiber were linear. In such a case, we have for $\phi_{0}=-\pi / 2$,

$$
\langle M\rangle \simeq 2 \sqrt{\bar{n}_{+} \bar{n}_{-}} \sin (2 \bar{N} \phi),
$$

and $\Delta^{2} M=\bar{N}$ for all $\phi$, so that after Eq. (2.10),

$$
\Delta^{2} \phi=\frac{1}{16 \bar{N} \bar{n}_{+} \bar{n}_{-} \cos (2 \bar{N} \phi)} .
$$

The optimum result holds for small enough signals $\phi \ll$ $1 / \bar{N}$ well below the Heisenberg limit of linear devices so that $\cos (2 \bar{N} \phi) \simeq 1$. When varying the balance of photons between the modes, the minimum uncertainty holds for an equal splitting of resources between the fiber modes, this is $\bar{n}_{+}=\bar{n}_{-}=\bar{N} / 2$ so that $|\psi\rangle=\left|\alpha_{1}=\sqrt{\bar{N}}\right\rangle\left|\alpha_{2}=0\right\rangle$ with a coherent state $\alpha=\sqrt{\bar{N}}$ in mode $a_{1}$ and vacuum in mode $a_{2}$. With all this we get

$$
\Delta^{2} \phi \simeq \frac{1}{4 \bar{N}^{3}}
$$

Note that is quite below the standard quantum limit and the Heisenberg limit of linear devices, so the result is consistent with the approximations made.

If we go beyond the simple estimation we have that the probe $\left|\alpha_{1}=\sqrt{\bar{N}}\right\rangle\left|\alpha_{2}=0\right\rangle$ has real coefficients in the number basis. So, if now we consider $\phi_{0}=0$, we get $F=F_{Q} \simeq 4 \bar{N}^{3}$ to the leading order in $\bar{N}$ for all $\phi$. As a bonus we get that the intense coherent states behave as minimum uncertainty states for the $G, M$ pair.

\section{B. Product of coherent and squeezed states}

The benefits of using squeezed states in linear quantum metrology are well-known from a long time ago [28-30]. We can check whether a similar result holds in the nonlinear case. To show this in the simplest manner we consider as probe state in mode $a_{1}$ a coherent state $|\alpha\rangle$ with real $\alpha$ and and squeezed vacuum in mode $a_{2}$ with squeezing parameter $r$ and mean number of photons $\bar{N}_{2}=\sinh ^{2} r$. Again we consider a fixed mean total number of photons $\bar{N}=|\alpha|^{2}+\bar{N}_{2}$ with $\bar{N} \gg 1$.

We begin with the simple evaluation of $\Delta \phi$ in Eq. (2.10) for $\phi_{0}=-\pi / 2$ and $\phi \rightarrow 0$. For the choice of the squeezing direction to reduce fluctuations of the quadrature $a_{2}+a_{2}^{\dagger}$ we have after Eq. (2.11),

$$
\Delta^{2} M=|\alpha|^{2} e^{-2 r}+\bar{N}_{2}
$$

and from Eq. (2.12)

$$
\langle[M, G]\rangle=2 i\left(|\alpha|^{4}+|\alpha|^{2}-\bar{N}_{2}^{2}-\Delta^{2} N_{2}\right),
$$

with

$$
\Delta^{2} N_{2}=2 \sinh ^{2} r \cosh ^{2} r .
$$

For $\alpha \gg 1$ and $r \gg 1$ it can be readily seen that the minimum $\Delta \phi$ in Eq. (2.10) holds for $\bar{N}_{2} \simeq \sqrt{\bar{N}} / 2 \ll \bar{N}$, leading to

$$
\Delta^{2} \phi=\frac{1}{4 \bar{N}^{7 / 2}} \text {. }
$$

Comparing Eqs. (3.5) and Eq. (3.9) we see that squeezing provides an effective improvement of resolution over the pure coherent case. Moreover, after noting that in these conditions $\Delta^{2} G \simeq 2 \bar{N}^{7 / 2}$ we get that the probe is close to be a minimum uncertainty states of the pair $G, M$ since $\Delta^{2} G \Delta^{2} M \simeq 2 \bar{N}^{4}$ is twice the minimum $|\langle[M, G]\rangle|^{2} / 4 \simeq \bar{N}^{4}$.

In Appendix A we have carried out an analysis of errors in the presence of several sources of noise that can be readily accounted for by the replacement in the detection operator $M$ in Eq. (2.11),

$$
a_{1}^{\dagger} a_{2} \rightarrow e^{i \varphi}\left(\sqrt{\eta} a_{1}^{\dagger}+\sqrt{1-\eta} b_{1}^{\dagger}\right)\left(\sqrt{\eta} a_{2}+\sqrt{1-\eta} b_{2}\right),
$$

where $\varphi$ is a random phase Gaussian distributed with zero mean and variance $\sigma^{2}, \eta$ is the quantum efficiency of the detectors, and $b_{j}$ are uncorrelated field modes in thermal states carrying $N_{t}$ photons with $N_{t} \ll \bar{N}$. The signal uncertainty becomes

$$
\Delta^{2} \phi \simeq \frac{1}{4 \eta^{2} \bar{N}^{7 / 2}}+\frac{N_{t}+1-\eta}{4 \eta^{3} \bar{N}^{3}}+\frac{\sigma^{2}}{2 \eta^{2} \bar{N}^{5 / 2}} .
$$

The dominant factors are the fluctuations of the relative phase $\varphi$ that would spoil the improvement caused by the squeezing vacuum unless $\sigma^{2}<1 / \bar{N}$. The other two terms would tend to phase uncertainty similar to the case of pure coherent probes unless $N_{t}+1-\eta<1 / \sqrt{\bar{N}}$.

After the tools in Sec. II E we can go beyond taking into account that when $\alpha$ is real and when the squeezing takes place either in the quadrature $i\left(a_{2}-a_{2}^{\dagger}\right)$ or in $a_{2}+a_{2}^{\dagger}$, the coefficients of the probe state in the number basis are real so that $F=F_{Q}$. Then we can ask for the balance between $\alpha^{2}$ and $\bar{N}_{2}$ that leads to maximum $F_{Q}$ at fixed $\alpha^{2}+\bar{N}_{2}=\bar{N}$. For $\alpha \gg 1$ we can neglect the fluctuations in mode $a_{1}$. Moreover, for $r \gg 1$ we can carry our the following approximation for the squeezed mode $N_{2} \simeq \bar{N}_{2} X^{2}$, where $X$ is a Gaussian variable with $\langle X\rangle=0$ and $\left\langle X^{2}\right\rangle=1$. In this limit it can be readily seen that the optimum result is $F_{Q} \simeq 30 \bar{N}^{4}$ that holds for $\bar{N}_{2} \simeq 0.71 \bar{N}$, so that for every $\phi$,

$$
\Delta^{2} \phi \simeq \frac{1}{30 \bar{N}^{4}} .
$$

Comparing Eqs. (3.9) and (3.12) we can appreciate that the resolution provided by the complete number statistics $p_{n}$ outperforms the simple estimation after the measurement of $M$. This can be regarded as a generalization to nonlinear interferometry of the result for the linear case in Ref. [30]. It is also worth noting that both in the linear and nonlinear schemes optimum results are obtained for the same probe states. The only difference is the amount of squeezing, $50 \%$ in 
the linear case versus $70 \%$ in the nonlinear one. This is to say that nonlinearity offers resolution improvement without any drawback in the probe preparation.

\section{Product of number states}

The above cases refer to realistic probe states. This can be compared with more ideal probes, such as the product of number states $|\psi\rangle=\left|n_{1}\right\rangle\left|n_{2}\right\rangle$ with $n_{1}+n_{2}=\bar{N}$. Starting with the simple estimator in Eq. (2.10) and using the complete exact expression Eq. (2.9), we get

$$
\langle M\rangle=\left(n_{1}-n_{2}\right) C_{1}
$$

and

$$
\left\langle M^{2}\right\rangle=\left(n_{1}-n_{2}\right) C_{1}^{2}+\left(2 n_{1} n_{2}+n_{1}+n_{2}\right) S_{1}^{2},
$$

where $S_{1}=\sin \left[2 \phi(\bar{N}-1)+\phi_{0}\right], \quad C_{1}=\cos [2 \phi(\bar{N}-1)+$ $\left.\phi_{0}\right]$, so that for any $\phi$ and $\phi_{0}$,

$$
\Delta^{2} \phi=\frac{2 n_{1} n_{2}+n_{1}+n_{2}}{4\left(n_{1}+n_{2}-1\right)^{2}\left(n_{1}-n_{2}\right)^{2}} .
$$

The minimum uncertainty holds either when $n_{1}=0$ or $n_{2}=$ 0 , so that for large $\bar{N}$ the uncertainty scales as

$$
\Delta^{2} \phi \simeq \frac{1}{4 \bar{N}^{3}} .
$$

This input probe is an $\mathrm{SU}(2)$ coherent state, which are the projection on fixed total number of the standard coherent states [31]. Accordingly, the resolution is the same reached with coherent probes in Eq. (3.5). However, note that in this case the result holds for all $\phi$ because here there is no dispersive effect caused by the fluctuations of $N$. Beyond this minimum, the uncertainty Eq. (3.15) grows without limit as $n_{1}$ approaches $n_{2}$. Deep down this holds because for $n_{1}=n_{2}$ we get that $\langle M\rangle$ no longer depends on the signal $\phi$.

Regarding the more advanced estimation provided by the complete statistics $p_{n}$, we get the opposite conclusions. After the Fisher information $F=F_{Q}=4\left(n_{1}+n_{2}\right)^{2}\left(2 n_{1} n_{2}+n_{1}+\right.$ $n_{2}$ ) the minimum uncertainty holds for $n_{1}$ as close as possible to $n_{2}$, this is $n_{1}=n_{2}=\bar{N} / 2$ for even $\bar{N}$, which is the well-known case of twin photon states [32]. This leads to $F_{Q} \simeq 2 \bar{N}^{4}$ and

$$
\Delta^{2} \phi \simeq \frac{1}{2 \bar{N}^{4}} .
$$

On the other hand, the minimum $F_{Q}$ holds for the SU(2) coherent states $n_{1} n_{2}=0$ with $F_{Q} \simeq 4 \bar{N}^{3}$ in agreement with Eq. (3.16).

In this particular case, it is possible to reach the optimum resolution Eq. (3.17) via the simple estimation procedure considering that the measured observable is $M^{2}$ instead of $M$. To simplify the calculation we consider from the start the probe with $n_{1}=n_{2}=\bar{N} / 2 \gg 1$ for even $\bar{N}$. In such a case,

$$
\left\langle M^{2}\right\rangle \simeq \frac{\bar{N}^{2}}{2} S_{1}^{2},
$$

and

$$
\Delta^{2} M^{2} \simeq S_{1}^{2}\left(\frac{\bar{N}^{4}}{8} S_{1}^{2}+2 \bar{N}^{2} C_{1}^{2}\right)
$$

Considering $\phi_{0}=0$ and $\phi \rightarrow 0$, we get, finally,

$$
\Delta^{2} \phi \simeq \frac{1}{2 \bar{N}^{4}},
$$

reaching the minimum value predicted by the Cramér-Rao bound in Eq. (3.17). Incidentally with the above computations it can be easily checked that the product of twin number states tend to be minimum uncertainty states of the pair $G, M^{2}$ for $\phi_{0}=0$ as $\phi \rightarrow 0$, this is $\Delta G \Delta M^{2} \simeq\left|\left\langle\left[G, M^{2}\right]\right\rangle\right| / 2$.

\section{CONCLUSIONS}

We have examined the performance of nonlinear gyroscopes in the quantum regime that highlight some relevant features for quantum metrology. This schemes does not imply length variations, so that this can be a built-in solid detector where all the potential advantages of nonlinearity can be used without the drawbacks caused if lengths were allowed to vary. Then the interferometer can be made of optical fibers where length and field confinement can be much improve the nonlinear effects.

A key result is that the optimum resolution can be approached by feasible coherent-squeezed inputs. This is a translation to nonlinear detection of the same result already proved for linear schemes. We find it remarkable that in this nonlinear scheme where the linear and nonlinear propagations commute the benefits of nonlinear schemes can be obtained for the same probe states of the linear interferometry. However, this may not be the case if the corresponding generators do not commute.

\section{ACKNOWLEDGMENTS}

We thank Prof. L. Pezzé for helpful comments. We acknowledge financial support from Spanish Ministerio de Economía y Competitividad Projects No. FIS2012-33152 and No. FIS2012-35583, and from the Comunidad Autónoma de Madrid research consortium QUITEMAD+ Grant No. S2013/ICE-2801.

\section{APPENDIX A: NOISE ANALYSIS}

For completeness let us address a simple but meaningful analysis of the effect of unavoidable sources of noise, such as finite quantum efficiency, thermalization, and phase randomization. To be more specific, we focus on the cases of pure coherent and coherent-squeezed probes since they provide the most interesting, meaningful, and practical of the situations studied above. Several sources of noise can be readily accounted for by the replacement in the detection operator $M$ in Eq. (2.11),

$$
a_{1}^{\dagger} a_{2} \rightarrow e^{i \varphi}\left(\sqrt{\eta} a_{1}^{\dagger}+\sqrt{1-\eta} b_{1}^{\dagger}\right)\left(\sqrt{\eta} a_{2}+\sqrt{1-\eta} b_{2}\right),
$$

where $\varphi$ is a random phase that we will assume to be Gaussian distributed with zero mean and variance $\sigma^{2}, \eta$ is the quantum efficiency of the detectors, and $b_{j}$ are uncorrelated field modes in thermal states with $\left\langle b_{1}\right\rangle=\left\langle b_{2}\right\rangle=\left\langle b_{1}^{\dagger} b_{2}\right\rangle=0$, and $(1-$ $\eta)\left\langle b_{1}^{\dagger} b_{1}\right\rangle=(1-\eta)\left\langle b_{2}^{\dagger} b_{2}\right\rangle=N_{t} / 2$ with $N_{t} \ll N$, respecting 
that quantum efficiency and thermalization are independent sources of uncertainty.

In such a case for probes in the product of coherent and squeezed states we have that in the absence of signal,

$$
\begin{aligned}
\Delta^{2} M= & \left\langle M^{2}\right\rangle=\eta^{2}\left(\alpha^{2}\left\langle a_{2}^{2}\right\rangle e^{-2 \sigma^{2}}\right. \\
& \left.+\alpha^{2}\left\langle a_{2}^{\dagger}\right\rangle e^{-2 \sigma^{2}}+\alpha^{2}\left\langle a_{2}^{\dagger} a_{2}\right\rangle+\left\langle a_{2}^{\dagger} a_{2}\right\rangle\right) \\
& +\eta\left(\alpha^{2}+\left\langle a_{2}^{\dagger} a_{2}\right\rangle\right)\left(N_{t}+1-\eta\right),
\end{aligned}
$$

where $\alpha$ is the coherent amplitude assumed real. Taking into account that in our case $\left\langle a_{2}^{2}\right\rangle=-\cosh r \sinh r,\left\langle a_{2}^{\dagger} a_{2}\right\rangle=$ $\sinh ^{2} r$, assuming $r \gg 1$, and considering the optimum case where the number of photons in the squeezed state is $\left\langle a_{2}^{\dagger} a_{2}\right\rangle=$ $\bar{N}_{2} \simeq \sqrt{\bar{N}} / 2 \ll \bar{N}$, we get

$$
\Delta^{2} M=\eta^{2} \sqrt{\bar{N}}+\eta^{2} \bar{N}^{3 / 2} \epsilon+\eta \bar{N}\left(N_{t}+1-\eta\right)
$$

where $\epsilon=1-\exp \left(-2 \sigma^{2}\right) \simeq 2 \sigma^{2}$, while for the denominator in Eq. (2.10) we have just $|\partial\langle M\rangle / \partial \phi| \simeq 2 \eta^{2} N^{2}$. Therefore, the final form for the signal uncertainty becomes

$$
\Delta^{2} \phi \simeq \frac{1}{4 \eta^{2} \bar{N}^{7 / 2}}+\frac{N_{t}+1-\eta}{4 \eta^{3} \bar{N}^{3}}+\frac{\epsilon}{4 \eta^{2} \bar{N}^{5 / 2}} .
$$

The most potentially harmful is the last term caused by the fluctuations of the relative phase $\varphi$. This is natural because variations of $\varphi$ cause that the coherent field couples with the antisqueezed component of the vacuum mode $a_{2}$, so the planned squeezing reduction becomes actually noise amplification. This effect would spoil the improvement caused by the squeezing vacuum unless $\epsilon \simeq 2 \sigma^{2} \leqslant 1 / \bar{N}$. The other two terms in $\Delta^{2} \phi$ are due to finite quantum efficiency and thermal photons, and would tend to phase uncertainty similar to the case of pure coherent probes unless $N_{t}+1-\eta \leqslant 1 / \sqrt{\bar{N}}$.

On the other hand, for the case of pure coherent probes we get that the uncertainty preserves the scaling,

$$
\Delta^{2} \phi \simeq \frac{N_{t}+1}{4 \eta^{3} \bar{N}^{3}}
$$

in accordance with the robustness of coherent light.

\section{APPENDIX B: LINEAR AND NONLINEAR PHASE SHIFTS}

A relevant characteristic of the propagation in optically nonlinear media is that the field experiences always both linear and nonlinear effects. This raises a very interesting question regarding which transformation will encode optimally the signal, and whether its performance would be affected by the presence of the other one. The proper arena to examine this issue is a multiparameter estimation procedure [23]. More specifically, if the signal-dependent transformation is

$$
U_{\Omega}=e^{-i \phi_{\mathrm{L}} G_{\mathrm{L}}-i \phi_{\mathrm{NL}} G_{\mathrm{NL}}},
$$

lower bounds for the estimation of $\phi_{\mathrm{L}}$ and $\phi_{\mathrm{NL}}$ can be obtained in terms of the quantum Fisher information matrix as

$$
F=4\left(\begin{array}{cc}
\Delta^{2} G_{\mathrm{L}} & \operatorname{Re}\left\langle G_{\mathrm{L}} G_{\mathrm{NL}}\right\rangle-\left\langle G_{\mathrm{L}}\right\rangle\left\langle G_{\mathrm{NL}}\right\rangle \\
\operatorname{Re}\left\langle G_{\mathrm{L}} G_{\mathrm{NL}}\right\rangle-\left\langle G_{\mathrm{L}}\right\rangle\left\langle G_{\mathrm{NL}}\right\rangle & \Delta^{2} G_{\mathrm{NL}}
\end{array}\right)
$$

as

$$
\Delta^{2} \phi_{\mathrm{L}} \geqslant\left(F^{-1}\right)_{1,1}, \quad \Delta^{2} \phi_{\mathrm{NL}} \geqslant\left(F^{-1}\right)_{2,2} .
$$

In our case we have

$$
G_{\mathrm{L}}=i\left(a_{2}^{\dagger} a_{1}-a_{1}^{\dagger} a_{2}\right), \quad G_{\mathrm{NL}}=N i\left(a_{2}^{\dagger} a_{1}-a_{1}^{\dagger} a_{2}\right),
$$

with $\left[G_{\mathrm{L}}, G_{\mathrm{NL}}\right]=0$. Since we are dealing with quantum Fisher information we may follow the same approximations leading to Eq. (3.12).

After an straightforward calculation we finally get that for fixed mean total photon number $\bar{N}$ the optimum $\Delta^{2} \phi_{\mathrm{L}}$ holds for $\bar{N}_{2} \simeq 0.6 \bar{N}$ leading to $\Delta^{2} \phi_{\mathrm{L}} \simeq 0.3 / \bar{N}^{2}$ which is rather close to the Heisenberg limit $0.25 / \bar{N}^{2}$.

On the other hand the optimum $\Delta^{2} \phi_{\mathrm{NL}}$ holds for $\bar{N}_{2} \simeq 0.8 \bar{N}$ leading to $\Delta^{2} \phi_{\mathrm{NL}} \simeq 0.04 / \bar{N}^{4}$ which is also rather close to
Eq. (3.12). Therefore, we may say that the multiparameter protocol reproduces expected results for the estimation of linear and nonlinear phase shifts which seem to be not affected by the presence of the other part.

We have carried out the same analysis for the case when there is no squeezing so the mode $a_{2}$ is in the plain vacuum state leading to, in the limit $\bar{N} \gg 1$, to $\Delta^{2} \phi_{\mathrm{L}} \simeq$ $1 / 4$ and $\Delta^{2} \phi_{\mathrm{NL}} \simeq 0.25 / \bar{N}^{2}$. So in comparison with the squeezed case the conclusion would be the opposite in the sense that there would be a clear perturbation between linear and nonlinear estimation processes, as already encountered in an slightly different single-mode nonlinear detector scheme [23].
[1] A. Luis, Nonlinear transformations and the Heisenberg limit, Phys. Lett. A 329, 8 (2004).

[2] S. Boixo, S. T. Flammia, C. M. Caves, and J. M. Geremia, Generalized Limits for Single-Parameter Quantum Estimation, Phys. Rev. Lett. 98, 090401 (2007).
[3] S. Boixo, A. Datta, M. J. Davis, S. T. Flammia, A. Shaji, and C. M. Caves, Quantum Metrology: Dynamics versus Entanglement, Phys. Rev. Lett. 101, 040403 (2008).

[4] S. Boixo, A. Datta, M. J. Davis, A. Shaji, A. B. Tacla, and C. M. Caves, Quantum-limited metrology and Bose-Einstein condensates, Phys. Rev. A 80, 032103 (2009). 
[5] C. Gross, T. Zibold, E. Nicklas, J. Estéve, and M. K. Oberthaler, Nonlinear atom interferometer surpasses classical precision limit, Nature 464, 1165 (2010).

[6] M. Napolitano and M. W. Mitchell, Nonlinear metrology with a quantum interface, New J. Phys. 12, 093016 (2010).

[7] A. B. Tacla, S. Boixo, A. Datta, A. Shaji, and C. M. Caves, Nonlinear interferometry with Bose-Einstein condensates, Phys. Rev. A 82, 053636 (2010).

[8] A. Rivas and A. Luis, Precision Quantum Metrology and Nonclassicality in Linear and Nonlinear Detection Schemes, Phys. Rev. Lett. 105, 010403 (2010).

[9] A. Luis, Quantum-limited metrology with nonlinear detection schemes, SPIE Reviews 1, 018006 (2010).

[10] M. Napolitano, M. Koschorreck, B. Dubost, N. Behbood, R. J. Sewell, and M. W. Mitchell, Interaction-based quantum metrology showing scaling beyond the Heisenberg limit, Nature 471, 486 (2011).

[11] R. J. Sewell, M. Napolitano, N. Behbood, G. Colangelo, F. Martin Ciurana, and M. W. Mitchell, Ultrasensitive Atomic Spin Measurements with a Nonlinear Interferometer, Phys. Rev. X 4, 021045 (2014).

[12] A. Luis and A. Rivas, Nonlinear Michelson interferometer for improved quantum metrology, Phys. Rev. A 92, 022104 (2015).

[13] X. Jin, M. Lebrat, L. Zhang, K. Lee, T. Bartley, M. Barbieri, J. Nunn, A. Datta, and I. A. Walmsley, Surpassing the Conventional Heisenberg Limit using Classical Resources, CLEO: QELS Fundamental Science 2013, San Jose, CA, OSA Technical Digest (online) (OSA, Washington, D.C., 2013).

[14] S. F. Huelga, C. Macchiavello, T. Pellizzari, A. K. Ekert, M. B. Plenio, and J. I. Cirac, Improvement of Frequency Standards with Quantum Entanglement, Phys. Rev. Lett. 79, 3865 (1997).

[15] R. Demkowicz-Dobrzański, K. Banaszek, and R. Schnabel, Fundamental quantum interferometry bound for the squeezedlight-enhanced gravitational wave detector GEO 600, Phys. Rev. A 88, 041802(R) (2013).

[16] R. Demkowicz-Dobrzański, M. Jarzyna, and J. Kołodyński, Quantum limits in optical interferometry, Prog. Opt. 60, 345 (2015).

[17] K. U. Schreiber and J.-P. R. Wells, Large ring lasers for rotation sensing, Rev. Sci. Instrum. 84, 041101 (2013).

[18] B. P. Abbott et al. (LIGO Scientific Collaboration and Virgo Collaboration), Observation of Gravitational Waves from a Binary Black Hole Merger, Phys. Rev. Lett. 116, 061102 (2016).

[19] M. O. Scully and M. S. Zubairy, Quantum Optics (Cambridge University Press, Cambridge, England, 1997).

[20] L. M. Rico-Gutierrez, T. P. Spiller, and J. A. Dunningham, Quantum-enhanced gyroscopy with rotating anisotropic Bose Einstein condensates, New J. Phys. 17, 043022 (2015).

[21] M. J. Woolley, G. J. Milburn, and C. M. Caves, Nonlinear quantum metrology using coupled nanomechanical resonators, New J. Phys. 10, 125018 (2008).

[22] C. González-Santander, F. Domínguez-Adame, and R. A. Römer, Excitonic Aharonov-Bohm effect in a two-dimensional quantum ring, Phys. Rev. B 84, 235103 (2011).

[23] J. Cheng, Quantum metrology for simultaneously estimating the linear and nonlinear phase shifts, Phys. Rev. A 90, 063838 (2014).
[24] M. Toren and Y. Ben-Aryeh, The problem of propagation in quantum optics, with applications to amplification, coupling of EM modes and distributed feedback lasers, Quantum Opt. 6, 425 (1994); J. Reháček, L. Mišta Jr., and J. Peřina, Codirectional simulation of contradirectional propagation, J. Mod. Opt. 46, 801 (1999); J. Peřina Jr. and J. Peřina, Quantum statistics of nonlinear optical couplers, Prog. Opt. 41, 361 (2000); A. Lukš and V. Peřinová, Canonical quantum description of light propagation in dielectric media, ibid. 43, 295 (2002).

[25] D. Maldonado-Mundo and A. Luis, Metrological resolution and minimum uncertainty states in linear and nonlinear signal detection schemes, Phys. Rev. A 80, 063811 (2009).

[26] C. W. Helstrom, Minimum mean-squared error of estimates in quantum statistics, Phys. Lett. A 25, 101 (1967); The minimum variance of estimates in quantum signal detection, IEEE Trans. Inform. Theory 14, 234 (1968); Quantum Detection and Estimation Theory (Academic, New York, 1976); S. L. Braunstein and C. M. Caves, Statistical Distance and the Geometry of Quantum States, Phys. Rev. Lett. 72, 3439 (1994).

[27] V. Peřinová, A. Lukš, and J. Křepelka, States of the optimum Fisher measure of information for quantum interferometry, Phys. Rev. A 73, 063807 (2006); G. A. Durkin and J. P. Dowling, Local and Global Distinguishability in Quantum Interferometry, Phys. Rev. Lett. 99, 070801 (2007).

[28] C. M. Caves, Quantum-mechanical noise in an interferometer, Phys. Rev. D 23, 1693 (1981); M. Xiao, L.-A. Wu, and H. J. Kimble, Precision Measurement Beyond the Shot-Noise Limit, Phys. Rev. Lett. 59, 278 (1987); P. Grangier, R. E. Slusher, B. Yurke, and A. LaPorta, Squeezed-Light-Enhanced Polarization Interferometer, ibid. 59, 2153 (1987); LIGO Scientific Collaboration, A gravitational wave observatory operating beyond the quantum shot-noise limit, Nat. Phys. 7, 962 (2011); Enhanced sensitivity of the LIGO gravitational wave detector by using squeezed states of light, Nat. Photon. 7, 613 (2013).

[29] A. Monras, Optimal phase measurements with pure Gaussian states, Phys. Rev. A 73, 033821 (2006); M. D. Lang and C. M. Caves, Optimal Quantum-Enhanced Interferometry using a Laser Power Source, Phys. Rev. Lett. 111, 173601 (2013).

[30] L. Pezzé and A. Smerzi, Mach-Zehnder Interferometry at the Heisenberg Limit with Coherent and Squeezed-Vacuum Light, Phys. Rev. Lett. 100, 073601 (2008).

[31] P. W. Atkins and J. C. Dobson, Angular momentum coherent states, Proc. R. Soc. London, Ser. A 321, 321 (1971).

[32] M. J. Holland and K. Burnett, Interferometric Detection of Optical Phase Shifts at the Heisenberg Limit, Phys. Rev. Lett. 71, 1355 (1993); T. Kim, J. Shin, Y. Ha, H. Kim, G. Park, T. G. Noh, and C. K. Hong, The phase-sensitivity of a MachZehnder interferometer for the Fock state inputs, Opt. Commun. 156, 37 (1998); H. Uys and P. Meystre, Quantum states for Heisenberglimited interferometry, Phys. Rev. A 76, 013804 (2007); F. W. Sun, B. H. Liu, Y. X. Gong, Y. F. Huang, Z. Y. Ou, and G. C. Guo, Experimental demonstration of phase measurement precision beating standard quantum limit by projection measurement, Europhys. Lett. 82, 24001 (2008). 